\title{
EchoGéo
}

$20 \mid 2012$

Des mobilités aux transports. Regards croisés en Afrique de l'ouest

\section{La carte et le territoire}

Jean-Louis Chaléard

\section{OpenEdition}

Journals

Édition électronique

URL : https://journals.openedition.org/echogeo/13128

DOI : 10.4000/echogeo. 13128

ISSN : 1963-1197

Éditeur

Pôle de recherche pour l'organisation et la diffusion de l'information géographique (CNRS UMR 8586)

Référence électronique

Jean-Louis Chaléard, «La carte et le territoire », EchoGéo [En ligne], 20 | 2012, mis en ligne le 13 juillet 2012, consulté le 10 août 2021. URL : http://journals.openedition.org/echogeo/13128 ; DOI : https:// doi.org/10.4000/echogeo.13128

Ce document a été généré automatiquement le 10 août 2021.

EchoGéo est mis à disposition selon les termes de la licence Creative Commons Attribution - Pas d'Utilisation Commerciale - Pas de Modification 4.0 International (CC BY-NC-ND) 


\title{
La carte et le territoire
}

\author{
Jean-Louis Chaléard
}

1 Dans cette nouvelle livraison d'ÉchoGéo deux thèmes majeurs apparaissent. Le premier aborde la géographie électorale à travers l'analyse de la carte électorale à laquelle est consacrée la rubrique Sur le métier. D’André Siegfried à la multiplication des travaux récents, Michel Bussi, coordinateur du dossier, revient sur l'histoire de ce document, instrument d'analyse de la situation politique d'un pays autant que de l'état de sa démocratie, et sur son renouveau actuel. Les entretiens avec deux professionnels, Jérôme Fourquet, de l'IFOP, et Georges-Antoine Strauch, président fondateur d'Articque, éditeur de logiciels de cartographie, sont l'occasion de s'interroger sur le rôle de la carte électorale aujourd'hui, son utilisation par les médias et le danger d'un usage sans réflexion de cet outil. Ce dossier, fidèle à l'esprit de la rubrique, permet de toucher à l'un des «métiers» qu'offre la géographie et de mettre en évidence la nécessité d'une réflexion géographique sur le monde contemporain.

2 Il est complété par plusieurs articles de la rubrique Sur le vif, puisque 3 des 4 textes parus depuis la précédente livraison d'échoGéo portent sur les résultats d'élections. Ces articles illustrent l'élargissement $\mathrm{du}$ champ d'analyse de la géographie électorale qu'évoque Michel Bussi dans sa présentation. Un article traite des élections présidentielles qui ont eu lieu au Pérou en juin 2011. Les auteurs, Evelyne Mesclier et Alexis Sierra, s'appuient abondamment sur les cartes pour mener leur étude. La cartographie des votes à deux échelles (celle des régions et celle des districts de la capitale, Lima) aide à analyser les résultats, à pénétrer le comportement des électeurs et nous introduit dans les rouages de la société et de l'économie péruviennes qui permettent de comprendre le rassemblement des intellectuels et des laissez pour compte du libéralisme sur la candidature d'Ollanta Humala. Les articles sur les élections législatives de Côte d'Ivoire et présidentielles de République Démocratique du Congo posent un autre problème, en raison de la contestation des scrutins, dans des pays où la démocratie est balbutiante. En Côte d'Ivoire, les législatives ont été boycottées par le FPI de l'ancien président Laurent Gbagbo. En RDC, le scrutin présidentiel est contesté en raison des «cafouillages» et «tripatouillages» de tous ordres que décrit Roland Pourtier. L'analyse des cartes, non seulement des résultats mais aussi des taux de participation, s'avère révélatrice de l'état des deux pays. En Côte d'Ivoire, Christian 
Bouquet et Irène Kassi-Djodjo y voient une nouvelle étape de la démocratie ivoirienne, un gage pour l'avenir, pour autant que le parti majoritaire ne cède pas aux facilités que lui procurent ses larges succès électoraux. Le bilan est plus critique en ce qui concerne la RDC, avec des taux de participations de plus de $100 \%$ dans certaines circonscriptions, ce qui conduit Roland Pourtier à s'interroger sur le bien-fondé des modalités de représentation populaire dans certains pays d'Afrique. La carte "miroir de la démocratie? » se demande Michel Bussi. Cela inclut l'antidémocratie aussi.

3 Le second thème majeur de cette livraison d'ÉchoGéo porte sur les mobilités et les transports en Afrique de l'ouest, dossier de la rubrique Sur le champ. Les articles réunis ici ont pour la majorité été présentés lors d'un atelier qui s'est tenu à Uppsala dans le cadre de la conférence européenne des études africaines en 2011. Comme pour le dossier du précédent numéro sur la mer et le littoral, constitué à partir de communications de Journées du Comité National français de Géographie, les textes n'ont pas été tous repris. Un autre s'y est ajouté. Mais ÉchoGéo a jugé utile de diffuser les principaux résultats d'une manifestation qui a réuni quelques uns des meilleurs spécialistes européens du thème. En outre, en ne s'attachant pas seulement aux transports mais aussi aux mobilités, Jérôme Lombard et Olivier Ninot, les coordonnateurs du dossier, ont voulu insister sur l'enchevêtrement des circulations, leurs influences réciproques, et combler des lacunes dans la recherche sur «l'Afrique mobile ", relativement peu étudiée.

4 Les articles réunis renvoient, selon les responsables du dossier, à trois dynamiques principales qui structurent le couple mobilité/transport en Afrique de l'ouest. La première porte sur les corridors, les ports et les grands opérateurs qui restructurent l'espace. Les deux articles de Jean Debrie et Foussata Dagnogo et al. mettent en évidence le poids croissant des grands groupes internationaux dans la logistique, et ses conséquences à petite comme à grande échelle, construisant de nouvelles inégalités territoriales. La seconde dynamique, abordée par Gina Porter et Benjamin Steck, a trait au rôle structurant des routes dans l'organisation de l'espace et la mobilité des populations. La route est plus souple que d'autres modes de transport et laisse plus de place aux mobilités locales. Pour autant, ses effets à certains égards ne sont pas moins excluant que ceux des grandes infrastructures ferroviaires et portuaires. La troisième dynamique renvoie aux nouvelles mobilités issues parfois d'initiatives des populations elles-mêmes. Ainsi, l'article de Lourdes Diaz Olvera et al. souligne la vitalité des conducteurs de mototaxis. Le texte de Michel Lesourd s'interroge de façon originale sur la mobilité des élites africaine. Dans tous les cas, des recompositions fortes apparaissent dans le contexte actuel de libéralisation et de mondialisation. Entre grands groupes internationaux, États, populations locales aux possibilités inégales, on peut s'interroger, avec les auteurs du dossier, sur les mutations à l'œuvre dans la gouvernance des transports. On rejoint alors des thèmes qui dépassent le cadre de l'Afrique et intéressent aussi bien les transports dans leur ensemble, que les effets des politiques publiques sur les territoires ou que le rôle de la mondialisation actuelle dans les processus de développement.

5 Ce dossier est complété par un carnet photographique qui permet d'illustrer la grande diversité des moyens de transport: mototaxis, minibus, minicars, charrettes, etc. Il donne à voir tout au long de la route, les multiples structures dont dépendent en partie la fluidité et l'importance des trafics : gares, marchés, barrages... Il se termine par la représentation de quelques uns des acteurs principaux, de la marchande au voyageur. 
Comme souvent, l'image ne fait pas qu'illustrer. En donnant à voir, elle permet de pousser l'analyse, d'insister sur certains détails, de faire vivre une réalité que le discours scientifique a parfois tendance à déshumaniser. Dans la rubrique Sur l'écrit, Henri Nicolaï nous offre une lecture croisée de deux manuels sur l'Afrique «noire » ou "subsaharienne", à l'occasion de leur récente réédition. Les auteurs des ouvrages, africanistes reconnus, proposent des visions parallèles, refusant l'afro-pessimisme ambiant sans ignorer les crises et les difficultés du sous-continent. Ce compte rendu permet de mettre en perspective le propos du dossier sur les transports et les mobilités, dont les dynamiques peuvent être intégrées à des processus plus larges touchant l'ensemble des espaces et des sociétés d'une Afrique plus que jamais plurielle et « ambiguë ».

6 Enfin, le dernier dossier de la rubrique Sur l'image est consacré aux représentations des enfants du Nord de la France sur la frontière. Au delà du thème retenu, il révèle le rôle du dessin comme outil méthodologique pertinent. À travers des interrogations sur l'image ou les représentations graphiques, on rejoint ici nos réflexions initiales sur la carte électorale. Comme le dessin des enfants, la carte est un instrument qui permet d'aller à la rencontre de la société et du territoire. Mais il s'agit d'un instrument scientifiquement élaboré. Contrairement à Jed Martin, le héros de Michel Houellebec, le géographe ne trouve pas la carte plus intéressante que le territoire. Si elle le passionne, c'est parce qu'elle est à la fois une construction intellectuelle et un indispensable outil d'analyse. 